\title{
Potentially Inappropriate Medication Use in Older Adults with Alzheimer's Disease and Related Dementias Living in the Community: A Cross-Sectional Analysis
}

\author{
Lauren E. Vickers $^{1} \cdot$ Ashley I. Martinez ${ }^{1} \cdot$ Alexandra M. Wallem $^{1} \cdot$ Clare Johnson $^{1} \cdot$ Daniela C. Moga $^{1,2,3,4}(\mathbb{D}$
}

Accepted: 22 May 2021 / Published online: 10 June 2021

(c) The Author(s) 2021

\begin{abstract}
Background With the older adult population in the USA increasing, so is the population of those with Alzheimer's disease and related dementias (ADRD). Older adults are vulnerable to the effects of potentially inappropriate medications as established by the Beers Criteria; however, some medications continue to be prescribed against recommendations.

Objectives Our objectives were to describe potentially inappropriate medication (PIM) use linked to cognitive impairment or decline (referred to as Cog-PIM) in older adults with and without ADRD and to investigate whether the odds of Cog-PIM report differ by ADRD status in ambulatory care (i.e., outpatient care) in the USA.

Methods A cross-sectional analysis was performed using a nationally representative sample of non-perioperative, officebased ambulatory care visits by adults aged $\geq 65$ years in $2016(n=218,182,131)$. Data were collected from the National Ambulatory Medical Care Survey. Cog-PIMs were identified as defined in the 2015 Beers Criteria recommendations for medications that may be potentially inappropriate in older adults with cognitive impairment or dementia. ADRD status was determined by clinician report using free text, the ADRD flag, or the presence of a diagnosis code indicating dementia. Multivariable logistic regressions were used to estimate the odds of Cog-PIM use overall and by medication class.

Results In 2016, 2.1\% ( $n=4,651,563)$ of outpatient visits were made by older adults with ADRD, 33.2\% of which reported at least one Cog-PIM. Anticholinergic Cog-PIMs were noted in 20.5\% of ADRD visits compared with $8.1 \%$ of non-ADRD visits. Antipsychotic PIMs were noted in $15.5 \%$ of ADRD visits compared with $0.8 \%$ of non-ADRD visits. Benzodiazepine and non-benzodiazepine receptor agonist hypnotic ( $\mathrm{Z}$ drug) Cog-PIMs were reported in 10.9\% of ADRD visits and $10.7 \%$ of non-ADRD visits. ADRD status was a significant predictor of Cog-PIM report overall (adjusted odds ratio [aOR] 2.74 [95\% confidence interval $\{\mathrm{CI}\}$ 1.20-6.27]) and for anticholinergics and antipsychotics specifically (aOR 3.35 [95\% CI 1.24-9.03] and aOR 22.80 [95\% CI 5.80-89.50], respectively).

Conclusion This study demonstrated a high prevalence of Cog-PIM use and increased odds of Cog-PIM use in older adults with ADRD. Future work should investigate opportunities in the ambulatory care setting for safer prescribing and deescalation of Cog-PIMs.
\end{abstract}

Daniela C. Moga

daniela.moga@uky.edu

Extended author information available on the last page of the article

\section{Key Points}

In a national sample of US ambulatory visits, approximately one-third of the patients with Alzheimer's disease and related dementia were using medications that could potentially have a negative impact on their cognition.

Identifying these medications during an ambulatory visit provides a good opportunity to consider deprescribing and treatment optimization to balance risks and benefits for individual patients. 


\section{Introduction}

The older adult population in the USA is growing disproportionately because of decreasing fertility rates and increasing life expectancy [1]. Within this population, the number living with Alzheimer's disease and related dementias (ADRD) is also increasing and is currently more than 5.8 million and expected to approach 14 million by 2050 [2]. The challenges associated with caring for this population continue to evolve as the number of patients being seen by primary care physicians grows [2], as does the frequency of medication-related visits [3].

Older adults with ADRD are particularly vulnerable to potentially inappropriate prescribing and the adverse effects of potentially inappropriate medication (PIM) use. PIMs are medications, both prescribed and non-prescribed, for which risks might outweigh benefits, so continued use needs to be evaluated. The vulnerability of this population is in part because of physiologic changes associated with aging that reduce renal and hepatic clearance of medications [4]. Additionally, given the burden of multimorbidity in the older adult population [5], indicated pharmacological treatment can increase the risk for polypharmacy (concurrent use of five or more medications) [6]. This is complicated further in the ADRD population by complex care needs, communication challenges and changing goals of care [4, $7,8]$. Patients with ADRD may have a variety of symptoms such as agitation, aggression, or sleep disturbances for which pharmacotherapy may seem an attractive treatment option, but the risks associated with many of these medications may outweigh their clinical benefit, leading organizations such as the American Geriatrics Society to recommend against their use [9-11].

Thus, identifying and avoiding PIMs when possible is essential in disease and symptom management in older adults. The Beers Criteria is a widely accepted and commonly used tool to identify PIMs for older adults, including those with ADRD [11, 12]. It specifies the following four medication classes known to exacerbate existing cognitive impairment or dementia (Cog-PIMs): anticholinergics, antipsychotics, benzodiazepines, and non-benzodiazepine receptor agonist hypnotics ( $\mathrm{Z}$ drugs). These medications are associated with significant adverse events in the ADRD population, including negative cognitive effects, falls, fractures, cardiovascular events, hospitalization, and increased mortality [13-15]. Despite recommendations against their use [11], these medications are commonly prescribed [16-18]. Previous studies of this nature have been limited to one medication class [19], were not specific to older adults with ADRD [20], used other PIM identification tools [21], or were conducted in international populations [22]. Therefore, this study investigated Cog-PIM use among patients with and without ADRD in a cross-sectional, nationally representative sample of US ambulatory care visits in 2016. Ambulatory care refers to medical services performed on an outpatient basis, without admission to a hospital or other facility and includes wellness, diagnosis, treatment, and rehabilitation visits.

\section{Methods}

\subsection{Data and Study Population}

We used data from the National Ambulatory Medical Care Survey (NAMCS) to conduct a cross-sectional study investigating whether older adults with ADRD reported different Cog-PIM use at outpatient visits compared with those without ADRD. NAMCS data are collected annually and are nationally representative of US physician visits in the ambulatory care setting after population-based weights are applied. The survey instrument collects data on patient demographics, provider characteristics, and visit details, including chronic conditions reported, tests and procedures provided, and medication use.

For the purposes of this study, we included visits made by patients aged $\geq 65$ years from 1 January 2016 through 31 December 2016, excluding perioperative visits because the nature of these visits may not represent chronic medication use. At the time this study was conducted, 2016 was the latest available data from NAMCS. The data are publicly available, de-identified, and do not directly involve human subjects, so this study was exempt from the institutional review board process at the University of Kentucky (IRB number 53287). Full details regarding the datasets, methods of collection and data maintenance, and survey samples can be found on the NAMCS website [23].

\subsection{Measures and Covariates}

The NAMCS data collection form allows for up to 30 medications to be recorded from the patient's health record. All the medications in the research analytical files are coded using the Lexicon Plus ${ }^{\circledR}$, a proprietary database of Cerner Multum, Inc. [23]. This study defined Cog-PIMs as in the 2015 update to the Beers Criteria based on information included in Tables 2 (2015 American Geriatrics Society Beers Criteria for Potentially Inappropriate Medication Use in Older Adults) and 3 (2015 American Geriatrics Society Beers Criteria for Potentially Inappropriate Medication Use in Older Adults Because of Drug-Disease or Drug-Syndrome Interactions That May Exacerbate the Disease or Syndrome), including anticholinergics, antipsychotics, benzodiazepines, and $\mathrm{Z}$ drugs [11]. Medications reported as new and continued were included and classified using the Lexicon 
Plus ${ }^{\circledR}$ nomenclature. Visits for patients with ADRD were identified from the data collection form using the free-text diagnosis fields provided by physicians, the binary indicator for Alzheimer's disease/dementia, and the presence of an International Classification of Diseases, Tenth Edition (ICD-10) code indicating dementia (G30.0, G30.1, G30.8, G309, F01.50, F01.51, F02.80, F02.81, F03.90, F03.91, F10.27, F10.97, F13.27, F13.97, F18.27, F18.97, F19.27, and F19.97). We identified demographic characteristics including female sex, age categories $(65-74,75-84$, and $\geq$ 85 years), and non-white race. Visit characteristics included indicators of first visit status, patient new to provider, patient seen by multiple providers, visit for a new problem, and visit lasting at least $30 \mathrm{~min}$. Clinical characteristics included report of tobacco use, cerebrovascular disease diagnosis, cancer diagnosis, depression diagnosis, number of comorbidities (chronic conditions), total medications, and report of polypharmacy (five or more medications).

\subsection{Data Analysis}

We examined differences in demographic and clinical characteristics by ADRD status using Chi squared tests for categorical variables; Student's $t$ tests for continuous, normally distributed variables; and Wilcoxon and Mann-Whitney $U$ tests for continuous, non-normally distributed variables.

Multivariable logistic regressions were used to estimate odds ratios (ORs) and 95\% confidence intervals (CIs) for report of any Cog-PIM use and use of anticholinergic, antipsychotic, and benzodiazepine/Z drug Cog-PIM use. Benzodiazepine and $\mathrm{Z}$ drug classes were combined because of small sample sizes and their closely related mechanisms of action. All regressions were adjusted for measured covariates likely to confound the relationship between Cog-PIM use and ADRD based on previous studies, as described.

We conducted all analyses in SAS 9.4 (SAS Institute, Cary, NC, USA).

\section{Results}

\subsection{Study Population Characteristics}

The 2016 NAMCS included 13,165 visits, of which 3888 met our inclusion criteria (we excluded 561 perioperative visits and 8716 visits for patients aged $<65$ years); after applying population-based weights, $218,182,131$ outpatient visits were analyzed (Table 1). Of these, approximately $2.1 \%(4,651,563$ weighted visits) reported ADRD as a patient diagnosis. ADRD visits were conducted more often by a primary care provider (62.6 vs. $35.8 \%$ ), were more often made by adults aged $\geq 75$ years ( 75.9 vs. $44.5 \%)$, and reported more chronic conditions (median 2.4 [interquartile range $\{$ IQR $\} 1.2-3.6$ ] vs. 1.7 [IQR 0.4-3.0]) and medications (median 6.1 [IQR 0.7-9.3] vs. 2.8 [IQR 0.1-7.1]). Of the specific chronic conditions, a diagnosis of depression was more frequently reported in patients with ADRD (25.3 vs. $9.6 \%$ ) (Table 1).

\subsection{Use of Potentially Inappropriate Medications Linked to Cognitive Impairment or Decline (Cog-PIM)}

Cog-PIM use was reported in $33.2 \%$ of visits for patients with ADRD compared with $17.0 \%$ of visits for patients without ADRD (see Table 1). Anticholinergic and antipsychotic medications were reported with greater frequency in ADRD visits ( 20.5 vs. $8.1 \%$ and 15.5 vs. $0.8 \%$, respectively). However, benzodiazepines and $\mathrm{Z}$ drugs did not show statistically significant differences in frequency reported between visits by ADRD status (8.7 vs. $8.8 \%$ and 2.2 vs. $1.9 \%$, respectively). A total of $22 \mathrm{Cog}$-PIMs were reported for patients with ADRD: anticholinergics (cyclobenzaprine, benztropine, meclizine, amitriptyline, dicyclomine, olanzapine, hydroxyzine, doxepin, oxybutynin, paroxetine, trifluoperazine, desipramine); antipsychotics (quetiapine, haloperidol, olanzapine, risperidone, trifluoperazine, aripiprazole); benzodiazepines (alprazolam, clonazepam, diazepam, lorazepam); and Z drugs (zolpidem, zaleplon).

At ADRD visits reporting an anticholinergic, the most commonly reported anticholinergic Cog-PIMs were cyclobenzaprine (25.3\%), benztropine (12.8\%), and meclizine (12.2\%). Cyclobenzaprine was more commonly reported in visits for patients with ADRD than with visits for patients without ADRD (25.3 vs. 12.5\%). Common antipsychotic Cog-PIMs at ADRD visits reporting this Cog-PIM class included quetiapine (40.0\%), haloperidol (26.2\%), and olanzapine (20.3\%). Haloperidol was reported at $26.2 \%$ of ADRD visits and was not reported at any non-ADRD visits. The most common benzodiazepine/Z drug Cog-PIMs for patients with ADRD were alprazolam (53.2\%), clonazepam (16.0\%), and zolpidem (13.5\%), with alprazolam being more common at ADRD visits (53.2 vs. $35.1 \%$ ).

After adjusting for potential confounders, visits for patients with ADRD had 2.74 times the odds of reporting Cog-PIM use than did visits for patients without ADRD (adjusted OR [aOR] 2.74 [95\% CI 1.20-6.27]; see Fig. 1). Visits for patients with ADRD also had higher odds of reporting anticholinergic and antipsychotic Cog-PIMs (aOR 3.35 [95\% CI 1.24-9.03] and aOR 22.80 [95\% CI $5.80-89.50]$, respectively). Reports of benzodiazepine or $\mathrm{Z}$ drug Cog-PIM use did not differ significantly based on ADRD status. 
Table 1 Baseline characteristics of older adults according to ADRD status in ambulatory care visits, 2016

\begin{tabular}{|c|c|c|c|c|}
\hline \multirow[t]{2}{*}{ Characteristics } & \multirow[t]{2}{*}{ Total } & \multicolumn{2}{|l|}{ ADRD status } & \multirow[t]{2}{*}{$p$ Value } \\
\hline & & Yes & No & \\
\hline \multicolumn{5}{|l|}{ Demographic characteristics } \\
\hline \multicolumn{5}{|l|}{ Sample size, $n$} \\
\hline Weighted visits & $218,182,131$ & $4,651,563$ & $213,530,568$ & \\
\hline Unweighted sample & 3888 & 73 & 3815 & \\
\hline Age, years, $\%$ & & & & 0.085 \\
\hline $65-74$ & 54.9 & 24.1 & 55.5 & \\
\hline $75-84$ & 32.9 & 47.0 & 32.7 & \\
\hline$\geq 85$ & 12.2 & 28.9 & 11.8 & \\
\hline Female sex, $\%$ & 56.5 & 60.1 & 56.5 & 0.645 \\
\hline Non-Hispanic white, $\%$ & 78.2 & 80.7 & 78.1 & 0.887 \\
\hline \multicolumn{5}{|l|}{ Clinical } \\
\hline New patient, $\%$ & 12.1 & 9.1 & 12.2 & 0.408 \\
\hline New reason for visit, $\%$ & 25.1 & 15.0 & 25.2 & 0.179 \\
\hline Length of visit, $\%$ & & & & 0.642 \\
\hline $0-14 \min$ & 14.9 & 11.4 & 15.0 & \\
\hline $15-29 \mathrm{~min}$ & 58.7 & 61.4 & 58.7 & \\
\hline $30-59 \mathrm{~min}$ & 24.2 & 22.5 & 24.2 & \\
\hline$\geq 60 \min$ & 2.2 & 4.7 & 2.1 & \\
\hline Seen by primary care provider, $\%$ & 36.4 & 62.6 & 35.8 & $<0.001$ \\
\hline Saw multiple providers, $\%$ & 50.5 & 55.1 & 50.4 & 0.600 \\
\hline Cancer diagnosis, $\%$ & 17.2 & 13.2 & 17.3 & 0.561 \\
\hline Cerebrovascular disease diagnosis, $\%$ & 4.9 & 5.6 & 4.9 & 0.813 \\
\hline Depression diagnosis, $\%$ & 9.9 & 25.3 & 9.6 & $<0.001$ \\
\hline Chronic conditions, median (IQR) & & $2.4(1.2-3.6)$ & $1.7(0.4-3.0)$ & \\
\hline Never tobacco user, $\%$ & 25.0 & 34.7 & 40.3 & 0.181 \\
\hline \multicolumn{5}{|l|}{ Medication } \\
\hline Five or more medications, $\%$ & 44.0 & 58.1 & 43.7 & 0.094 \\
\hline Number of medications, median (IQR) & & $6.1(0.7-9.3)$ & $2.8(0.1-7.7)$ & \\
\hline Any Cog-PIM, $\%$ & 17.3 & 33.2 & 17.0 & 0.005 \\
\hline Visits reporting any anticholinergic, $\%$ & 8.4 & 20.5 & 8.1 & 0.003 \\
\hline Visits reporting any antipsychotic, $\%$ & 1.1 & 15.5 & 0.8 & $<0.001$ \\
\hline Visits reporting any benzodiazepine, $\%$ & 8.8 & 8.7 & 8.8 & 0.977 \\
\hline Visits reporting any $\mathrm{Z}$ drug, $\%$ & 1.9 & 2.1 & 1.9 & 0.855 \\
\hline
\end{tabular}

$A D R D$ Alzheimer's disease and related dementias, Cog-PIM PIM linked to cognitive impairment or decline, $I Q R$ interquartile range, PIM potentially inappropriate medications, $Z$ drug non-benzodiazepine receptor agonist hypnotics

\section{Discussion}

In this pharmacoepidemiologic study, we investigated the relationship between ADRD status and Cog-PIM use in adults aged $\geq 65$ years in a sample of ambulatory care visits in the USA in 2016. The majority of patients with ADRD were seen by primary care providers, a characteristic similarly noted in a recent study of Medicare beneficiaries with ADRD [24]. The mean number of chronic conditions was higher in patients with ADRD, which is also consistent with previous literature findings [7]. Specifically, approximately one-quarter of patients with ADRD reported depression diagnosis, for which Cog-PIMs may be used in treatment. Depression in patients with ADRD is consistently listed as a comorbidity across the literature, with varying rates. A recent meta-analysis including clinicand community-based studies from all around the world found a mean prevalence of depression in ADRD of $42 \%$, with high heterogeneity between studies, as reflected by the wide range of depression prevalence of 19-78\% [25]. Our results represent outpatient visits in the URSA, with results similar to estimates coming from other communitybased studies conducted in the USA that were included in the meta-analysis. 


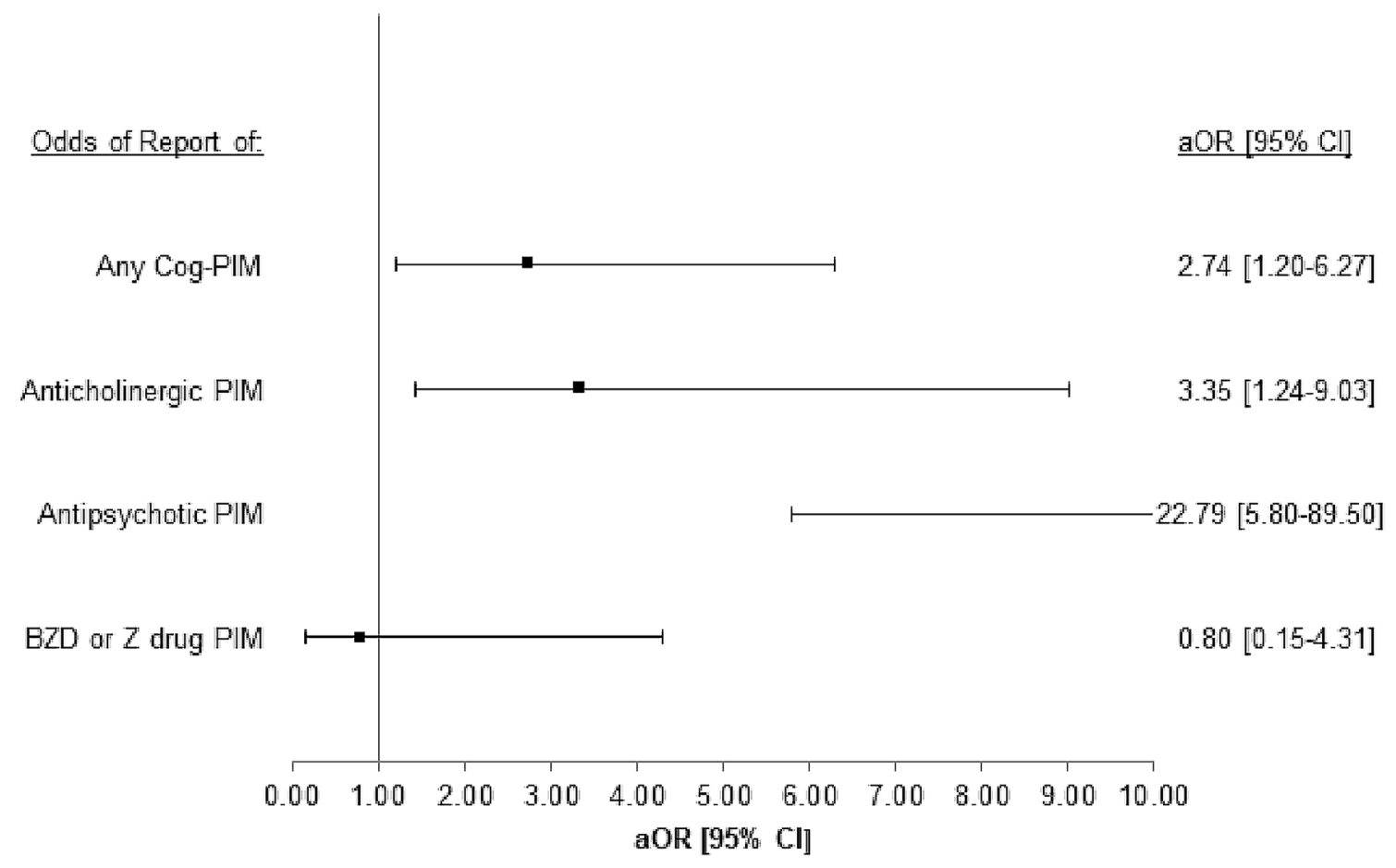

Fig. 1 Adjusted odds ratios of Cog-PIM report according to Alzheimer's disease and related dementias status in older adults in the ambulatory care setting, 2016. $a O R$ adjusted odds ratio, BZD benzo-

The main objective of this analysis was to determine the rate of Cog-PIM prescribing in older adults with ADRD. Our data indicated that, on average, approximately twice as many medications were reported at visits for patients with ADRD than at visits for patients without ADRD. Cog-PIMs were listed in one-third of ADRD visits, and polypharmacy was reported in more than half of ADRD visits. Few studies exist for comparison; however, one Danish study found polypharmacy reported for 63\% of its ADRD cohort, and 24-45\% reported PIM use, depending on the PIM identification tool [16]. A recent Australian study reported Cog-PIM use in $37 \%$ of its ADRD cohort, with anticholinergics and sedatives being the most common [22]. A study using a representative sample of the US population reported a prevalence of $26.95 \%$ for potentially inappropriate anticholinergic medication use among older adults with dementia [26]. Although higher Cog-PIM prevalence rates were identified in multiple studies, the main driving factors remain unclear. Patients with ADRD likely have multiple comorbid conditions, increasing the risk for polypharmacy, drug-drug and drug-disease interactions resulting in PIM, including Cog-PIM use. The complexity of care for patients with ADRD poses significant challenges in optimizing pharmacological treatments, avoiding undertreatment, and balancing risks and benefits.

Of the specific Cog-PIM classes, anticholinergics were most prevalent in ADRD visits, followed by antipsychotics. diazepine, $C I$ confidence interval, $C o g$-PIM potentially inappropriate medication linked to cognitive impairment or decline, $Z$ drug nonbenzodiazepine hypnotic

ADRD status was associated with greater odds of Cog-PIM use from both classes, driven primarily by antipsychotics. While ADRD status was not significantly associated with odds of benzodiazepine/Z drug Cog-PIM use, the frequency of report regardless of ADRD status was still high. Pharmacotherapy with Cog-PIMs for dementia-related behavioral symptom control is not recommended, and the Beers Criteria provide alternative therapy options for each Cog-PIM class [27]. Specifically, although atypical antipsychotics are recommended over typical antipsychotics for last-line, short-term use, this study demonstrated that haloperidol (a typical antipsychotic carrying a black box warning specific to this population) was among the most commonly reported antipsychotic in the Cog-PIM class [28]. Similarly, benzodiazepine use is not indicated for first-line or chronic use in patients with ADRD [29]. If necessary, short-acting benzodiazepines are preferred, yet only longer-acting benzodiazepines were reported at ambulatory care visits for older adults in the 2016 NAMCS survey. Finally, Z drugs are not recommended at all for treatment of sleep-related symptoms in patients with ADRD [30]. Although pharmacotherapy is generally only indicated for short-term use after exhausting other treatment options, this study demonstrates common use. These findings clearly indicate that opportunities exist for reducing PIM use and polypharmacy through deprescribing and de-escalation of Cog-PIMs or replacement with 
safer alternatives. Pharmacists, as medication experts, are well positioned to guide efforts to improve medication use in patients with ADRD through comprehensive medication reviews, medication therapy management, recommendations for safer alternatives, or deprescribing. Pharmacist-led deprescribing and treatment optimization interventions in this setting have been shown to be effective for replacement or discontinuation of inappropriate medications and dose reduction of PIMs [31, 32]. Furthermore, collaborative care practices that include pharmacists on the team are a successful model for chronic disease management in outpatient care and could be considered for implementation in the care of patients living with ADRD [33].

There are limitations to this study. The NAMCS database is limited in data collection to randomly selected visits during a 1-week period. Data are de-identified, which does mean that more than one visit could be attributed to the same patient, although this is unlikely. The survey does not capture hospital-related visits, so medication information from those encounters is excluded. Since only medications associated with the visits are captured, medication and chart information may be incomplete. These may all contribute to underreporting of complete medication lists. The study design was cross-sectional, so no inferences about trends may be made. Despite these limitations, the study is strengthened by the generalizability provided by the nationally representative dataset and the design evaluating all CogPIM classes simultaneously.

\section{Conclusion}

In a national sample of US ambulatory visits, Cog-PIMs were reported in approximately one-third of ADRD patient visits. Anticholinergics and antipsychotics were the most frequently reported Cog-PIMs and were associated with ADRD status. These medications are not recommended for routine use in older adults and patients with mild cognitive impairment or ADRD. Given the risks associated with these medications, future work should investigate interventions to deprescribe Cog-PIM use in the ADRD population. Pharmacists are ideally placed to make a difference and assure optimal treatment regimens for patients living with ADRD. Our study was conducted using a representative sample of the US population, but our results raise awareness of an important issue that would be of interest for patients with ADRD and their care providers worldwide.

\section{Declarations}

Funding Support for this study was provided by the National Institutes of Health/National Institute on Aging (R01 AG054130) (DM).
Conflicts of interest Lauren E Vickers, Ashley I Martinez, Alexandra M Wallem, Clare Johnson, and Daniela C Moga have no conflicts of interest that are directly relevant to the content of this article.

Ethics approval The data are publicly available, de-identified, and do not directly involve human subjects so were exempt from the institutional review board process at the University of Kentucky (IRB number 53287).

Consent to participate Not applicable

Consent for publication Not applicable

Availability of Data and Material The datasets generated and/or analyzed during the current study are available in the National Ambulatory Medical Care Survey (NAMCS) repository (https://www.cdc.gov/nchs/ ahcd/datasets_documentation_related.htm).

Code availability We conducted all analyses in SAS 9.4 (SAS Institute, Cary, NC).

Author Contributions Vickers, Moga: study concept and design, data analysis and interpretation, and preparation of manuscript. Martinez, Wallem, Johnson: supervision and revision of study concept and design, data analysis and interpretation, and preparation of manuscript.

Open Access This article is licensed under a Creative Commons Attribution-NonCommercial 4.0 International License, which permits any non-commercial use, sharing, adaptation, distribution and reproduction in any medium or format, as long as you give appropriate credit to the original author(s) and the source, provide a link to the Creative Commons licence, and indicate if changes were made. The images or other third party material in this article are included in the article's Creative Commons licence, unless indicated otherwise in a credit line to the material. If material is not included in the article's Creative Commons licence and your intended use is not permitted by statutory regulation or exceeds the permitted use, you will need to obtain permission directly from the copyright holder. To view a copy of this licence, visit http://creativecommons.org/licenses/by-nc/4.0/.

\section{References}

1. Roberts AW, Ogunwole SU, Blakeslee L, Rabe MA. The Population 65 Years and Older in the United States: 2016, American Community Survey Reports, ACS-38, U.S. Census Bureau, Washington, DC, 2018

2. Alzheimer's Association. 2020 Alzheimer's Facts and Figures. https://www.alz.org/media/Documents/alzheimers-facts-and-figur es.pdf. Accessed 12 Apr 2020

3. Maust DT, Blow FC, Wiechers IR, Kales HC, Marcus SC. National trends in antidepressant, benzodiazepine, and other sedative-hypnotic treatment of older adults in psychiatric and primary care. J Clin Psychiatry. 2017;78(04):e363-71. https://doi.org/10. 4088/JCP.16m10713.

4. Mangoni AA, Jackson SHD. Age-related changes in pharmacokinetics and pharmacodynamics: basic principles and practical applications: age-related changes in pharmacokinetics and pharmacodynamics. Br J Clin Pharmacol. 2003;57(1):6-14. https:// doi.org/10.1046/j.1365-2125.2003.02007.x.

5. Goodman RA, Ling SM, Briss PA, Parrish RG, Salive ME, Finke BS. Multimorbidity patterns in the United States: implications 
for research and clinical practice. J Gerontol A Biol Sci Med Sci. 2016;71(2):215-20. https://doi.org/10.1093/gerona/glv199.

6. Klotz U. Pharmacokinetics and drug metabolism in the elderly. Drug Metab Rev. 2009;41(2):67-76. https://doi.org/10.1080/ 03602530902722679

7. Clague F, Mercer SW, McLean G, Reynish E, Guthrie B. Comorbidity and polypharmacy in people with dementia: insights from a large, population-based cross-sectional analysis of primary care data. Age Ageing. 2016. https://doi.org/10.1093/ageing/afw176.

8. Andersen F, Viitanen M, Halvorsen DS, Straume B, Engstad TA. Co-morbidity and drug treatment in Alzheimer's disease: a cross sectional study of participants in the Dementia Study in Northern Norway. BMC Geriatr. 2011;11(1):58. https://doi.org/10.1186/ 1471-2318-11-58.

9. Gitlin LN, Kales HC, Lyketsos CG. Nonpharmacologic management of behavioral symptoms in dementia. JAMA. 2012;308(19):2020. https://doi.org/10.1001/jama.2012.36918.

10. Bloom HG, Ahmed I, Alessi CA, et al. Evidence-based recommendations for the assessment and management of sleep disorders in older persons. J Am Geriatr Soc. 2009;57(5):761-89. https:// doi.org/10.1111/j.1532-5415.2009.02220.x.

11. By the American Geriatrics Society 2015 Beers Criteria Update Expert Panel. American Geriatrics Society 2015 updated beers criteria for potentially inappropriate medication use in older adults. J Am Geriatr Soc. 2015;63(11):2227-46. https://doi.org/ 10.1111/jgs.13702.

12. Hukins D, Macleod U, Boland JW. Identifying potentially inappropriate prescribing in older people with dementia: a systematic review. Eur J Clin Pharmacol. 2019;75(4):467-81. https://doi.org/ 10.1007/s00228-018-02612-x.

13. Mintzer J, Burns A. Anticholinergic side-effects of drugs in elderly people. J R Soc Med. 2000;93(9):457-62. https://doi.org/ 10.1177/014107680009300903.

14. Markota M, Rummans TA, Bostwick JM, Lapid MI. Benzodiazepine use in older adults: dangers, management, and alternative therapies. Mayo Clin Proc. 2016;91(11):1632-9. https://doi.org/ 10.1016/j.mayocp.2016.07.024.

15. Leon C, Gerretsen P, Uchida H, Suzuki T, Rajji T, Mamo DC. Sensitivity to antipsychotic drugs in older adults. Curr Psychiatry Rep. 2010;12(1):28-33. https://doi.org/10.1007/ s11920-009-0080-3.

16. Kristensen RU, Nørgaard A, Jensen-Dahm C, Gasse C, Wimberley T, Waldemar G. Polypharmacy and potentially inappropriate medication in people with dementia: a nationwide study. J Alzheimers Dis. 2018;63(1):383-94. https://doi.org/10.3233/JAD-170905.

17. Eshetie TC, Nguyen TA, Gillam MH, Ellett LMK. Potentially inappropriate prescribing in people with dementia: an Australian population-based study. Int J Geriatr Psychiatry. 2019;34(10):1498-505. https://doi.org/10.1002/gps.5160.

18. Patel T, Slonim K, Lee L. Use of potentially inappropriate medications among ambulatory home-dwelling elderly patients with dementia: a review of the literature. Can Pharm J Rev Pharm Can. 2017;150(3):169-83. https://doi.org/10.1177/1715163517701770.

19. Eichler T, Wucherer D, Thyrian JR, et al. Antipsychotic drug treatment in ambulatory dementia care: prevalence and correlates. $\mathrm{J}$ Alzheimers Dis. 2014;43(4):1303-11. https://doi.org/10.3233/ JAD-141554.

20. Rhee TG, Choi YC, Ouellet GM, Ross JS. National prescribing trends for high-risk anticholinergic medications in older adults. J Am Geriatr Soc. 2018;66(7):1382-7. https://doi.org/10.1111/jgs.15357.
21. Barry HE, Cooper JA, Ryan C, et al. Potentially inappropriate prescribing among people with dementia in primary care: a retrospective cross-sectional study using the enhanced prescribing database. J Alzheimers Dis. 2016;52(4):1503-13. https://doi.org/ 10.3233/JAD-151177.

22. Cross AJ, George J, Woodward MC, et al. Potentially inappropriate medication, anticholinergic burden, and mortality in people attending memory clinics. J Alzheimers Dis. 2017;60(2):349-58. https://doi.org/10.3233/JAD-170265.

23. NAMCS/NHAMCS - Questionnaires, datasets, and related documentation. Published March 4, 2019. https://www.cdc.gov/nchs/ ahcd/ahcd_questionnaires.htm. Accessed 10 Apr 2020

24. Yang M, Chang C-H, Carmichael D, Oh ES, Bynum JPW. Who is providing the predominant care for older adults with dementia? J Am Med Dir Assoc. 2016;17(9):802-6. https://doi.org/10.1016/j. jamda.2016.04.026.

25. Zhao Q-F, Tan L, Wang H-F, et al. The prevalence of neuropsychiatric symptoms in Alzheimer's disease: systematic review and meta-analysis. J Affect Disord. 2016;190:264-71. https://doi.org/ 10.1016/j.jad.2015.09.069.

26. Kachru N, Carnahan RM, Johnson ML, Aparasu RR. Potentially inappropriate anticholinergic medication use in older adults with dementia. J Am Pharm Assoc (2003). 2015;55(6):603-12. https:// doi.org/10.1331/JAPhA.2015.14288 (PMID: 26501745).

27. Hanlon JT, Semla TP, Schmader KE. Alternative medications for medications in the use of high-risk medications in the elderly and potentially harmful drug-disease interactions in the elderly quality measures. J Am Geriatr Soc. 2015;63(12):e8-18. https://doi.org/ 10.1111/jgs.13807.

28. American Psychiatric Association. The American Psychiatric Association practice guideline on the use of antipsychotics to treat agitation or psychosis in patients with dementia. American Psychiatric Association; 2016. https://doi.org/10.1176/appi.books. 9780890426807

29. Borson S, Raskind MA. Clinical features and pharmacologic treatment of behavioral symptoms of Alzheimer's disease. Neurology. 1997;48(Issue 5, Supplement 6):17S-24S. https://doi.org/10.1212/ WNL.48.5_Suppl_6.17S.

30. Yesavage JA, Friedman L, Ancoli-Israel S, et al. Development of diagnostic criteria for defining sleep disturbance in Alzheimer's disease. J Geriatr Psychiatry Neurol. 2003;16(3):131-9. https:// doi.org/10.1177/0891988703255684.

31. Ammerman CA, Simpkins BA, Warman N, Downs TN. Potentially inappropriate medications in older adults: deprescribing with a clinical pharmacist: CPS impact on inappropriate medication burden. J Am Geriatr Soc. 2019;67(1):115-8. https://doi.org/ 10.1111 /jgs. 15623.

32. Kachru N, Carnahan RM, Johnson ML, Aparasu RR. Potentially inappropriate anticholinergic medication use in community-dwelling older adults: a national cross-sectional study. Drugs Aging. 2015;32(5):379-89. https://doi.org/10.1007/s40266-015-0257-x (PMID: 25832970).

33. Dills H, Shah K, Messinger-Rapport B, Bradford K, Syed Q. Deprescribing medications for chronic diseases management in primary care settings: a systematic review of randomized controlled trials. J Am Med Dir Assoc. 2018;19(11):923-935.e2. https://doi.org/10.1016/j.jamda.2018.06.021 (Epub 2018 Aug 11 PMID: 30108032). 


\section{Authors and Affiliations}

\section{Lauren E. Vickers $^{1} \cdot$ Ashley I. Martinez ${ }^{1}$ - Alexandra M. Wallem ${ }^{1} \cdot$ Clare Johnson $^{1} \cdot$ Daniela C. Moga ${ }^{1,2,3,4} \mathbb{C D}^{\circ}$}

Lauren E. Vickers

lauren.vickers@uky.edu

Ashley I. Martinez

ashleyirene.martinez@gmail.com

Alexandra M. Wallem

awallem@rx.umaryland.edu

Clare Johnson

cmjo268@uky.edu
1 University of Kentucky College of Pharmacy, Lexington, KY, USA

2 University of Kentucky College of Public Health, Lexington, KY, USA

3 University of Kentucky Institute for Pharmaceutical Outcomes and Policy, Lexington, KY, USA

4 Sanders-Brown Center on Aging, Lexington, KY, USA 